\title{
Perioperative management of von Willebrand patients at the time of implant placement: Case report
}

\author{
Hiroyuki Takashima ${ }^{1}$, Aiji Sato $(\text { Boku })^{2}$, Hironori Miiyamoto ${ }^{1}$, Shinichiro Kato ${ }^{1}$, Shota \\ Furuno $^{1}$, and Yasuyuki Shibuya ${ }^{1}$ \\ ${ }^{1}$ Nagoya City University Graduate School of Medical Sciences and Medical School \\ ${ }^{2}$ Aichi Gakuin University School of Dentistry
}

June 17, 2021

\begin{abstract}
von Willebrand disease is a hereditary disease associated with a tendency to bleed and it is important to reduce the possibility of bleeding in a procedure. This case describes the use of a hemostatic factor, Factor VIII concentrate (Confact F®), during a dental procedure involving teeth extraction and implant placement.
\end{abstract}

Title: Perioperative management of von Willebrand patients at the time of implant placement: Case report Authors: Hiroyuki Takashima D.D.S. ${ }^{1}$, Aiji Sato D.D.S., Ph.D. ${ }^{2}$, Hironori Miyamoro D.D.S., Ph.D. ${ }^{1}$, Shinichiro Kato D.D.S., Ph.D. ${ }^{1}$, Shota Furuno D.D.S. ${ }^{1}$, Yasuyuki Shibuya D.D.S., Ph.D. ${ }^{1}$,

Institutional affiliations and address for each author

${ }^{1}$ Department of Oral and Maxillofacial Surgery, Nagoya City University Graduate School of Medical Sciences

${ }^{2}$ Department of Anesthesiology, Aichi Gakuin University School of Dentistry

\section{Corresponding author}

Aiji Sato (Boku), Department of Anesthesiology, Aichi Gakuin University School of Dentistry 2-11 Suemoridori, Chikusaku, Nagoya 464-8651, Japan

Tel: +81-52-759-2102, Fax; +81-52-759-2102, Email: bokuaiji@dpc.aichi-gakuin.ac.jp

\section{Key Clinical Message}

In the management of patients with type 1 VWD, supplementation with Con Facto F® as well as hemodynamic stabilization with appropriate analgesia and sedation may be important to reduce the risk of bleeding.

\section{ABSTRACT}

von Willebrand disease is a hereditary disease associated with a tendency to bleed and it is important to reduce the possibility of bleeding in a procedure. This case describes the use of a hemostatic factor, Factor VIII concentrate (Confact F®), during a dental procedure involving teeth extraction and implant placement.

Keywords: , von Willebrand disease, a hemostatic factor, Factor VIII concentrate (Confact F®)

Introduction 
Eric von Willebrand first reported Von Willebrand disease (VWD) in $1926 .{ }^{1}$ It is a congenital condition that results in an excessive tendency to bleed owing to the quantitative and qualitative abnormalities of von Willebrand factor (VWF). ${ }^{2}$ In the perioperative management of VWD patients, it is necessary to reduce bleeding by preoperatively replenishing coagulation factors and stabilizing hemodynamics during anesthesia. Herein, we report our experience with a patient with VWD. We performed safe perioperative hemostatic management under intravenous sedation by supplementing with heat-treated factor VIII concentrate (Confact $\mathrm{F}^{\circledR}$ ) before extracting the teeth and placing implants. Written consent was obtained from the patient for this report.

\section{Case Report}

The patient is a 57 -year-old man (height, $173.5 \mathrm{~cm}$; weight, $61.7 \mathrm{~kg}$ ), and the

remaining teeth in his lower jaw were 1,2 , and 3 on the right side and 1,4 , and 5 on the left side. The patient had chronic apical periodontitis and severe periodontal disease. There was teeth movement, and it was difficult to preserve them. We planned to extract these teeth and place six implants (Figure 1).

A physician had suspected the presence of VWD when the patient was aged 21 years, but no detailed testing or treatment was performed. The family history was not relevant and the patient was on no current medications.

In terms of preoperative chest findings, the chest X-ray was normal (Figure 2) with a normal cardiothoracic ratio of $42 \%$, and there were no particular issues. Blood test results are shown in Table 1 . The platelet count, a hemostatic factor, was $360,000 / \mu l$, the activated partial thromboplastin time was 35.4 seconds, and the prothrombin time 11.7 seconds; all were within normal limits. However, coagulation factor VIII was $49 \%$, and VWF activity was $24 \%$, indicating that the coagulation factor was lower than normal. Based on detailed testing carried out by our hospital's hematology department, the patient was diagnosed with Type 1 VWD, in which there is a quantitative decrease in the VWF.

There was nothing of particular note in terms of family history. Implants in the upper jaw had already been placed at a different hospital, and there had been some difficulty stopping the bleeding when the teeth were extracted. In this case, after consulting with the hematology department, we decided that surgery would be possible by transfusing heat-treated factor VIII concentrate (Confact $\mathrm{F}^{\circledR}$ ), which contains VWF. This transfusion was done to prevent abnormal bleeding during surgery. Furthermore, considering the risk of bleeding due to fluctuations in hemodynamics, with the patient's consent, we planned to carry out tooth extraction and implant placement under local anesthesia with intravenous sedation.

\section{Anesthetic Course}

One hour before entering the operating room, 1,500 units of Confact $\mathrm{F}^{\circledR}$ were transfused. In terms of transfusion volume, although we planned to carry out implant placement, it was determined that the amount of bleeding would not differ greatly from that of tooth extraction alone, so we used $25 \mathrm{IU} / \mathrm{kg}$, basing our calculations on those published in the New England Journal of Medicine (Table 2). ${ }^{3}$ Immediately before surgery, we took another blood sample. We confirmed that VWF activity was $127 \%$ and that coagulation factor VIII had increased to $104 \%$. We then proceeded with the surgery. Considering that fluctuations in hemodynamics during surgery promote bleeding, intravenous sedation was performed to stabilize circulation. Oxygen (2 l/min) was administered transnasally, and cefmetazole sodium ( $1 \mathrm{~g})$ was administered preoperatively to prevent infection. During the operation, we used midazolam iv and propofol (1\% Diprivan Injection kit ${ }^{\circledR}$ ) target-controlled infusion for continuous intravenous sedation. Also, $2 \%$ Xylocaine Dental ${ }^{\circledR}$ with epinephrine 1:80,000 was used for local anesthesia. The operation time was 3 hours and 37 minutes, the anesthesia time was 4 hours and 16 minutes, and blood loss was $405 \mathrm{ml}$. Although it was a little difficult to stop bleeding during the operation, we completed the procedure with no major problems. No postoperative complications, such as bleeding were observed, and the prognosis was favorable. One week after the operation, evaluation of the coagulation factor VIII and VWF activity revealed that they had decreased to $48 \%$ and $23 \%$, respectively, which were close to their preoperative values (Table 3 ). 


\section{Discussion}

VWD is a congenital bleeding disorder inherited in an autosomal dominant manner. This condition consists of a quantitative or qualitative abnormality in the VWF, a hemostatic factor that causes a temporary hemostatic disorder. The VWF functions as an intrinsic coagulation factor that mediates platelet adhesion to subepithelial connective tissue and stabilizes binding to coagulation factor VIII.

A lack or decrease in these functions can cause bleeding. Under the disease classification proposed by the International Society on Thrombosis and Haemostasis, there are the following types of VWD: 1, 2A, 2B, 2M, $2 \mathrm{~N}$, and $3 .{ }^{4}$ Type 1 is the most common and features a quantitative deficiency of the VWF but no functional problems. It was previously reported that desmopressin (DDAVP) is effective for hemostasis management in mild to moderate hemophilia and VWD. ${ }^{5}$ Therefore, DDAVP was used in many cases considering side effects such as the risk of infection from hepatitis virus and AIDS. However, the increase in deficiency factor activity is uncertain. Although it is used for short-term hemostasis, it has limited application since it cannot be repeatedly or continuously used. The improved viral inactivation accuracy of Confact $\mathrm{F}^{\circledR}$ improves the purity and safety of the drug, and the risk of infection is said to be lower than before. ${ }^{6}$ Although in this case, we planned to perform tooth extraction and implant placement, it was determined that the amount of bleeding would not differ greatly from carrying out tooth extraction alone, so for the transfusion volume, we used $25 \mathrm{IU} / \mathrm{kg}$, basing our calculations on those published in the New England Journal of Medicine (Table 2 ). Although no abnormal bleeding was observed during or after surgery, it was necessary to pay attention to bleeding when carrying out implant placement. Furthermore, stabilizing hemodynamics using appropriate analgesia and sedation is also considered extremely important for reducing the risk of bleeding.

\section{Conclusion}

In this case, we carried out safe perioperative management under intravenous sedation combined with local anesthesia after preoperatively supplementing with heat-treated factor VIII concentrate (Confact $\mathrm{F}^{\circledR}$ ) when extracting teeth and placing implants for a Type 1 VWD patient. Stabilizing hemodynamics using appropriate analgesia and sedation is also considered important for reducing the risk of bleeding.

\section{Acknowlegements}

Not applicable

\section{Funding}

None

\section{Conflict of interest}

The authors declare that we have no conflict of interest.

\section{Data availability statement}

Not applicable due to patient privacy concerns.

\section{Author's contributions}

All authors contributed to the case. Preoperative management and anesthetic planning were performed by HT, SF and AS. Anesthetic Management was performed by AS, SK and HM. The first draft of the manuscript was written by AS and all authors commented on previous versions of the manuscript. YS helped with the supervision of the manuscript and development of the overall periopertative plan. All authors read and approved the final manscript.

\section{Ethical approval}

Not applicable.

\section{Consent for publication}


The authors obtained written consent for publication from the patient.

ORCID Aiji Sato (Boku) 0000-0003-0182-2543

\section{References}

1. von Willebrand, EA. 1926. Hereditar Pseudohemophili, Finska Lakaresallskpets Handlinger 68:87-112.

2. Nishi H, Miyauchi M, Takechi M, et al. 2007. Dental management for von

Willebrand disease patients who had difficulty in communication. Pediatric Oral and Maxillofacial Surgery. 1991;17:101-107.

3. Leebeek FW, Eikenboom JC. Von Willebrand's disease. New England Journal of Medicine. 2016;375:2067-2080.

4. Matsushita T. Hemophilia and von Willebrand disease - Focus on replacement therapy. Journal of the Japanese Society of Internal Medicine. 2009;98:58-68.

5. Yamamoto K, Matsuzuka Y, Morimoto Y, et al. Hemostatic effect of DDAVP on tooth

extraction in patients with mild and moderate hemophilia A and von Willebrand

disease. Japanese Journal of Oral and Maxillofacial Surgery. 1995;41:984-990.

6. Uda A, Jinno J, Kobayashi R, et al. Tooth extraction in sisters with von Willebrand disease. Nihon University Journal of Oral Science. 2004;30:129-133.

\section{Figure Legends}

Figure1

The remaining teeth in his lower jaw were 1,2, and 3 on the right side and 1, 4, and 5 on the left side. The patient had chronic apical periodontitis and severe periodontal disease.

Figure 2

The chest X-ray was normal with a normal cardiothoracic ratio of $42 \%$

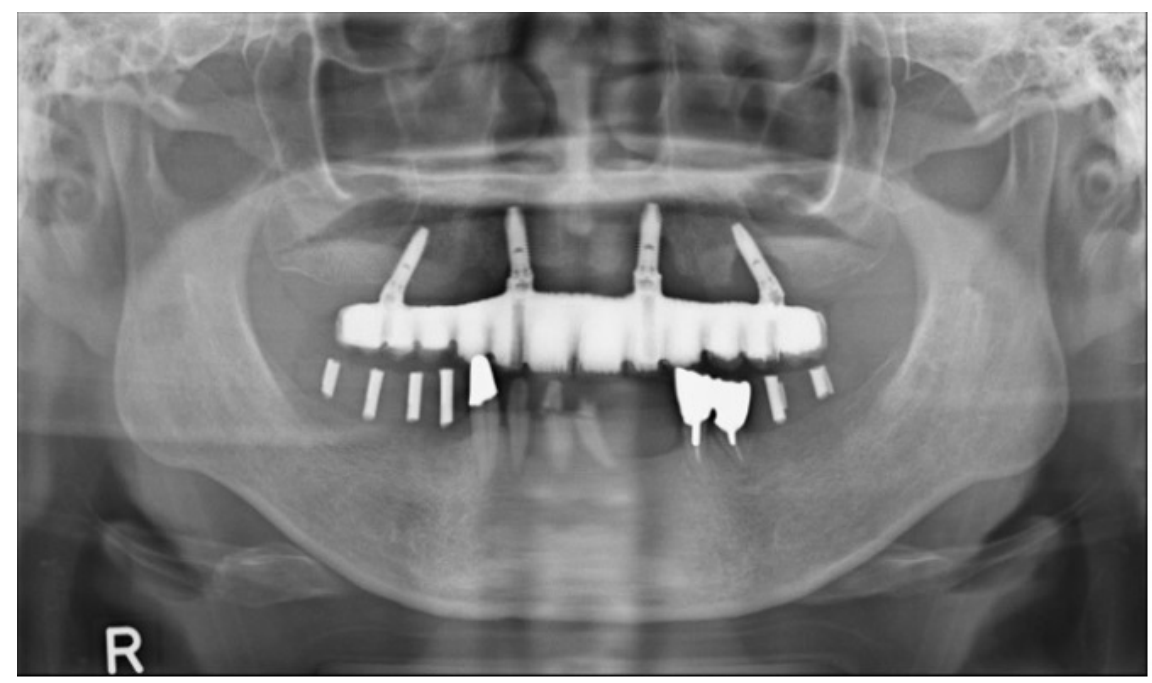




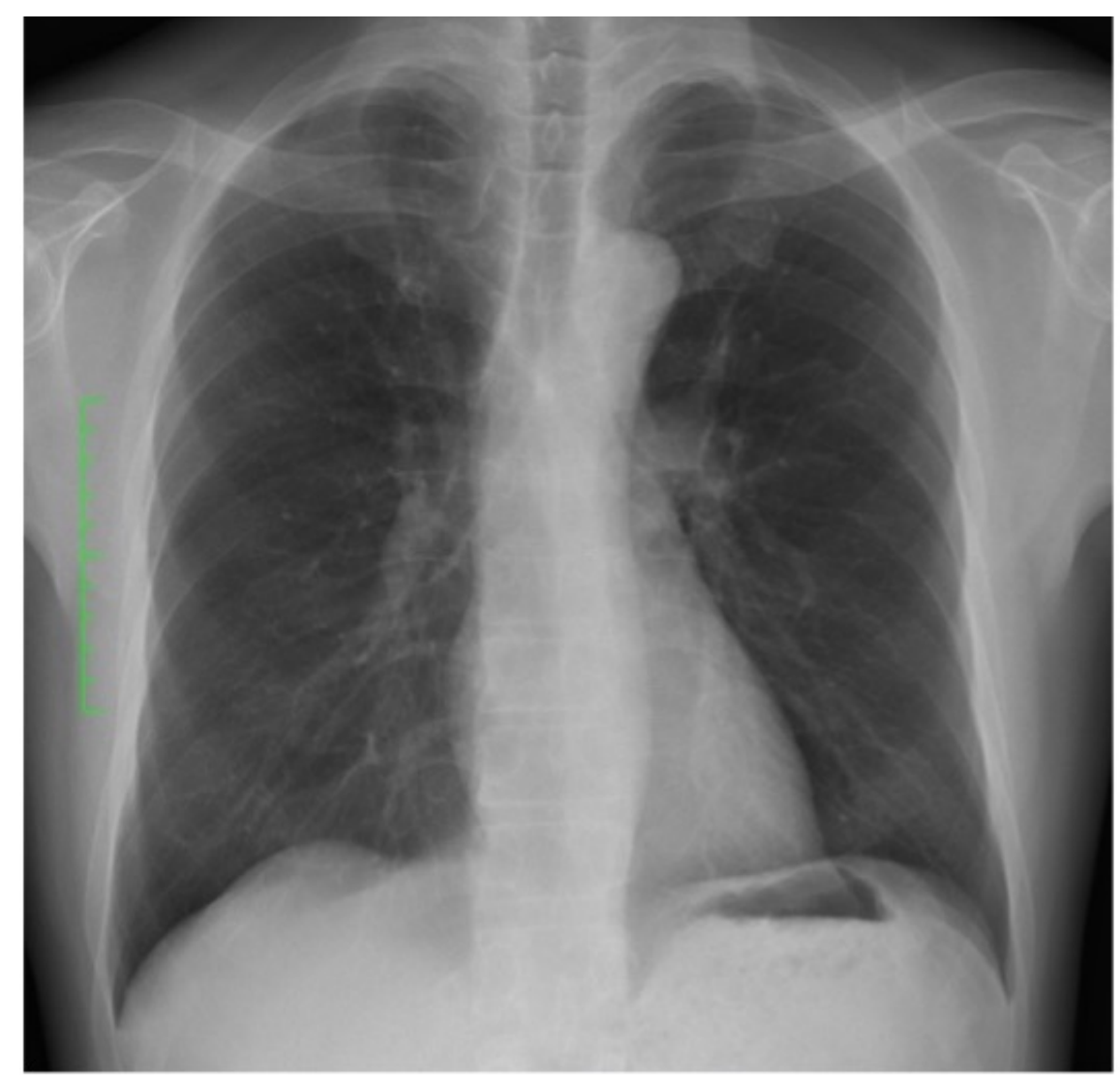

